\title{
pH Sensitive Lipid-Polymer Hybrid Nanoparticles Mediated Delivery of Docetaxel: A Viable Approach for Breast Cancer Therapeutic Intervention Development
}

\section{Ye Yuan}

Jilin University First Hospital

Jia-Xing Song

Jilin University First Hospital

Mei-Na Zhang

Jilin University first hospital

Baoshan Yuan ( $\sim$ baoshan_yuan@aliyun.com )

Jilin University First Hospital

Research

Keywords: Breast Cancer, pHS-LPHNPs-DTX, Docetaxel, cytosolic delivery, apoptosis

Posted Date: July 2nd, 2020

DOI: https://doi.org/10.21203/rs.3.rs-23464/v2

License: (c) (i) This work is licensed under a Creative Commons Attribution 4.0 International License.

Read Full License 
The authors have withdrawn this preprint from Research Square 